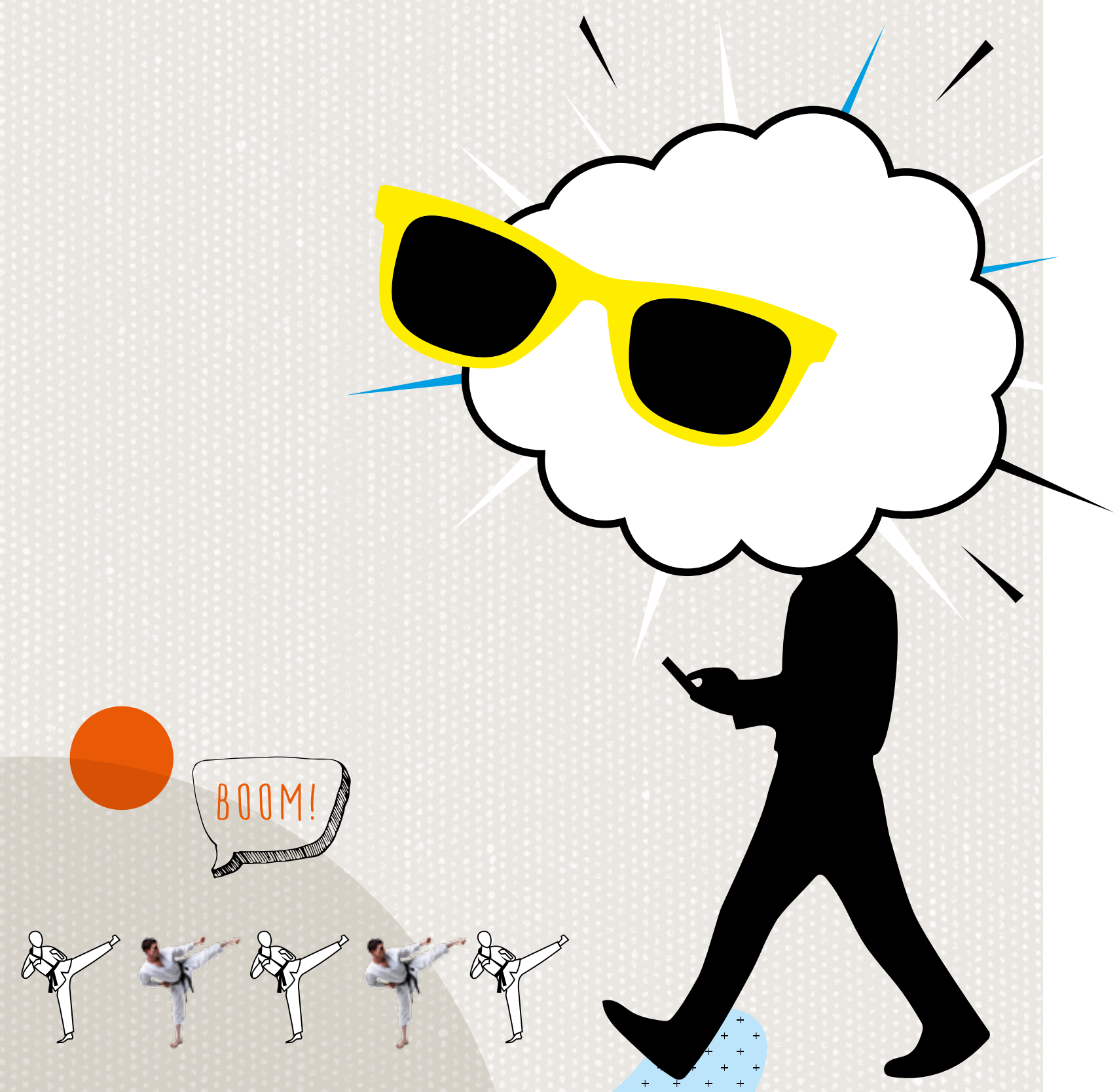




\title{
Are You Too Successful to Digitalize? How to Fight Innovation Blindness
}

\author{
Andreas Neus, Fabian Buder and Fernando Galdino
}

KEYWORDS

Innovation Blindness, Digitalization, Digital Transformation, Business Models, Mental Models

THE AUTHORS

Andreas Neus,

Head of Future and University Programs, andreas.neus@gfk-verein.org

Fabian Buder,

Project Manager - Future and University Programs fabian.buder@gfk-verein.org

Fernando Galdino,

Senior Trend Researcher - Future and University Programs fernando.galdino@gfk-verein.org

all: GfK Verein, Nuremberg, Germany
The digital turmoil /// Digitalization is often thought of as a technological challenge, and a seemingly rational response is to hire people with IT and data skills to deal with it. But while it is indeed important to ensure that a company's processes and systems are supported by state-of-the-art information technology, focusing only on this can lead to a false sense of security. The digital revolution is not only changing the way products and services are produced and consumed, but it is also fundamentally changing their perceived value and the competition in an increasingly transparent market. The whole market environment is changing: category boundaries erode, entry barriers are reduced, networks emerge, customers turn into competitors and everything happens much faster. Your company will be affected unless you have a legally guaranteed monopoly - and perhaps even then.

From technology to a digital mindset /// What was once considered expertise in the analog world might become a liability in the digital age instead of an asset. Established mental models can sometimes prevent a timely response to digital challenges. Digital transformation requires a digital mindset and new approaches to dealing with both decision risk and decision speed. If we compare the marketplace to a game of chess, then, until recently the players could assume that everybody played by the same basic rules and moved around the same set of pieces. In the digital era, the playing field has become much larger and new pieces have entered the scene, forming new alliances and trying out new moves. Companies that attempt to play the traditional game are in trouble as it is no longer possible to succeed with the old strategies (see Figure 1). 
FIGURE 1:

\section{If new players enter a market and change the rules of a game, it is no longer possible to succeed with the old strategies}

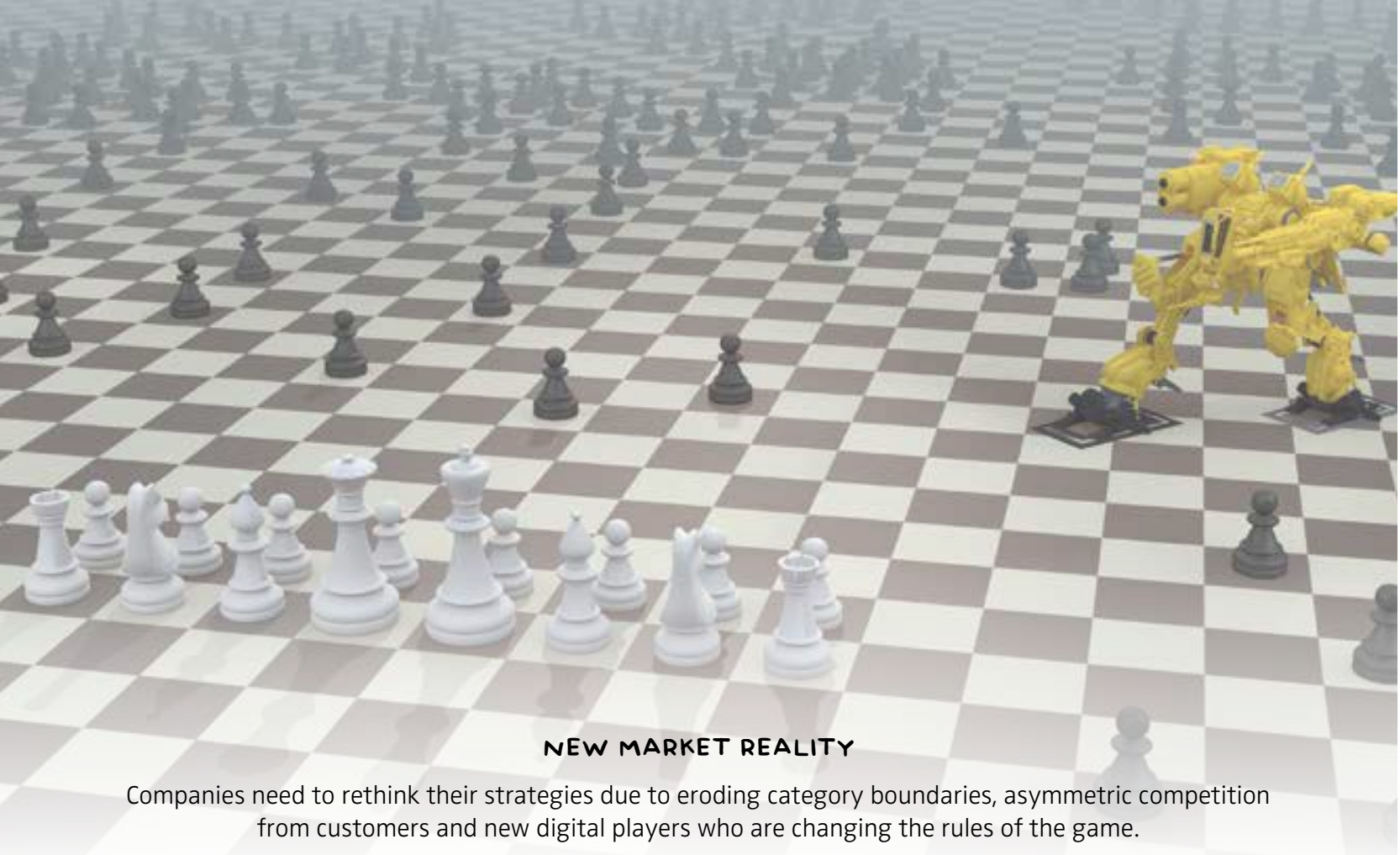

The interesting question is why many traditional companies just don't see what seems pretty obvious: that the game being played is no longer chess. Why are they unable to adapt their mindset, their rules of play to the new realities? What makes them act like a deer caught in the headlights instead of being innovative?

Causes of innovation blindness /// Well-entrenched companies seem to hold all the assets needed to adapt and keep succeeding: They have means to attract top talent and should be able analyze their market environment in time to detect and respond to changes. But sometimes even successful companies don't seem to adequately react to a disruptive threat; instead they show symptoms of what we call "innovation blindness." Their blind spots can often be attributed to the following phenomena.

> Overestimating one's own digital skills: the DunningKruger Effect /// Many top executives do not have a true digital mindset, and it may be that they are systematically overestimating their companies' digital competencies. The psychologists Dunning and Kruger found that those highly competent in a task have a good understanding of the limits of their skill and tend to underestimate their abilities. Contrarily, those who are incompetent in a task lack basic insight into how little they know and can vastly overestimate their own skill.

> Functional fixedness and mental models /// "Functional fixedness" is when our mental models concerning a problem or a solution are so engrained that we fail to recognize other perspectives or other applications. This is especially dangerous in a rapidly changing market environment. Deep expertise that used to be an asset turns into a liability: Improving push-button-phone functionality won't help against smart phones.

$>$ Sunk cost fallacy - reluctance to kill doomed projects early enough /// When companies stubbornly throw "good money after bad" in the hope to turn a failing pro- 
ject around, it can be due to a combination of the sunk cost fallacy and a reluctance to document failure. Ironically, if a company culture is too focused on "perfection" - rather than learning from small failures - it can end up systematically rewarding large failures by hiding troubled projects as long as possible. With regard to digitalization, learning happens too slowly and resources are being wasted.

\section{The end state of innovation blindness: the zombie orga-}

nization /// We call the end state of an organization that is unable to read the signs of its market a "zombie organization." A zombie organization has stopped responding to market feedback. It simply seems to keep doing what it has always done, even though outside observers can see the market no longer values the product or service the zombie organization traditionally offered. It behaves like an organism whose higher cognitive functions have been paralyzed but whose brain stem is still keeping basic functions operational. Once you have reached that state, it will be hard to interest digital talent in the company, and a successful digitalization becomes very unlikely.

\section{Sometimes even successful companies don't seem to adequately react to a disruptive threat; instead they show symptoms of what we call "innovation blindness."}

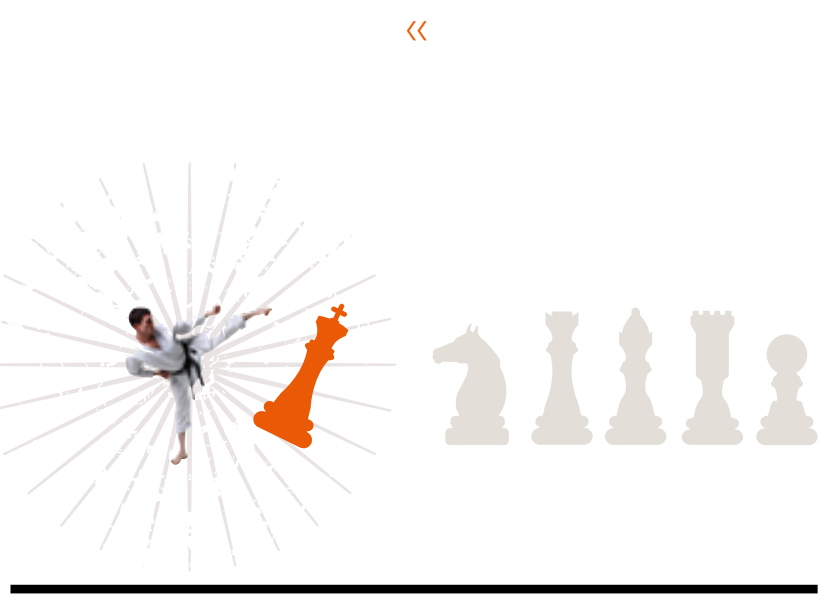

FIGURE 2:

Unchecked assumptions might be hazardous to your business model

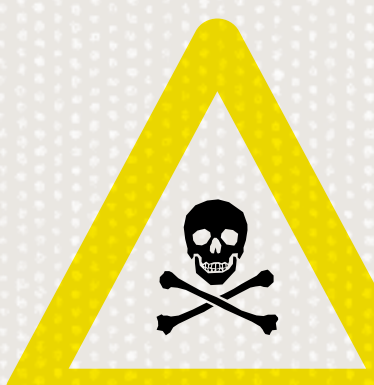

SURELY ALARM

- Surely we fully understand what our clients value us for ...

- Surely people cannot beat our quality, especially not at a lower price point ...

- Surely new entrants in the market first have to catch up before they can overtake us...

- Surely our customers will not be able to do this themselves ...

- Surely we understand new technology well enough to do our own digitalization ...

- Surely we would know if there was an existential threat to our business...

USE THE "SURELY ALARM" TO CHECK ASSUMPTIONS ABOUT YOUR MARKET, YOUR CUSTOMERS, YOUR ADDED VALUE AND YOUR DIGITAL SKILLS. 


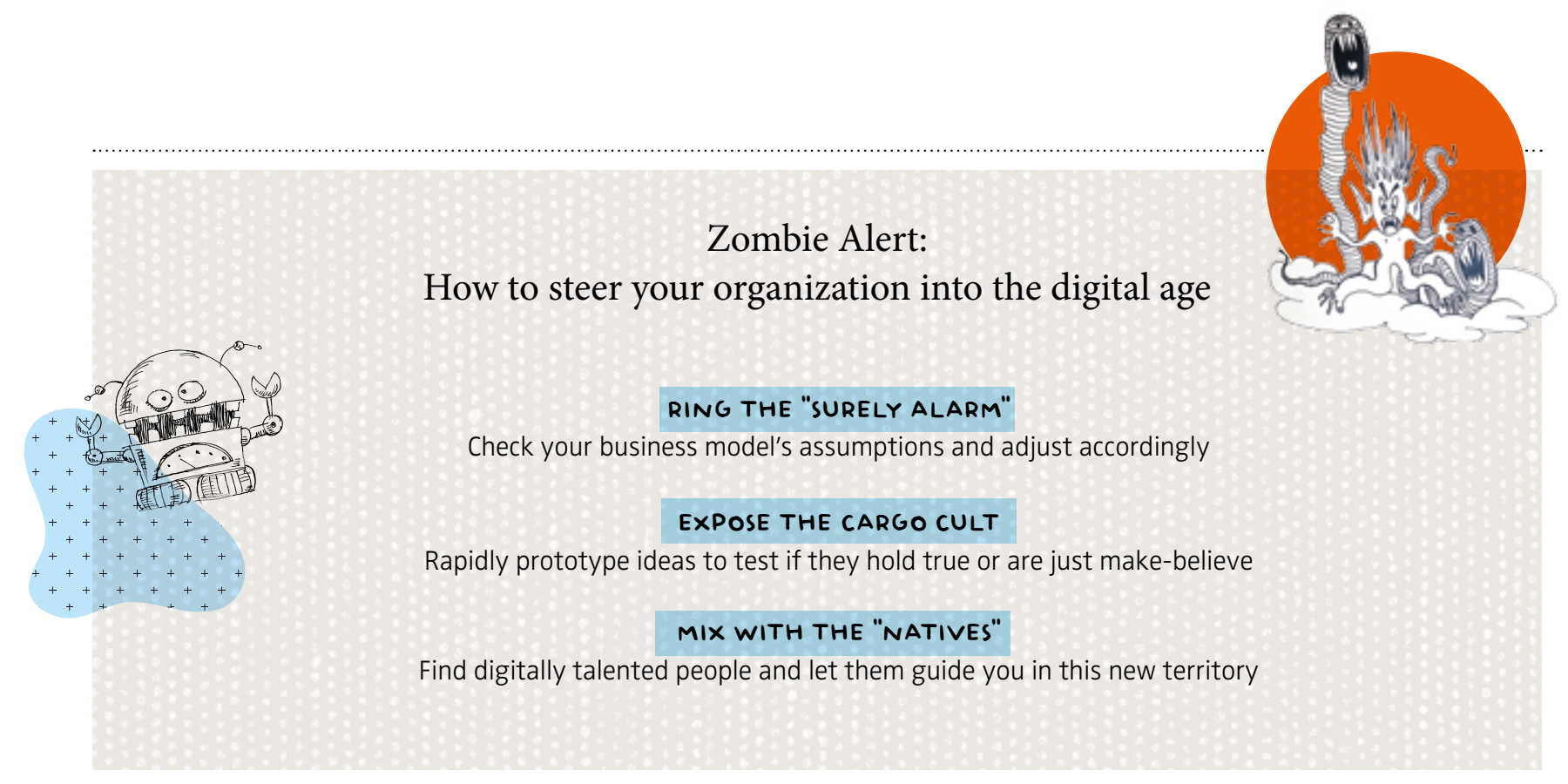

Fighting innovation blindness /// Innovation blindness is caused by holding onto outdated mental models and assumptions about how the world works. There are, however, a few things that can be done proactively to avoid major pitfalls in the digitalization of a business.

> Challenge your business model's basic assumptions with the "surely alarm" /// Business models are built on assumptions about customer wants and needs, unique value propositions, core competencies, market mechanisms, etc. Often the assumptions most fundamental to a business model are not recognized as assumptions but treated as irrefutable facts: "This is just the way our industry works." It is precisely these underlying assumptions that may be challenged by disruptive competitors who use digital tools to their full extent. Therefore seemingly given facts need to be challenged and checked against reality or empirical evidence to identify strategic failure in time.

To catch decision biases due to untested assumptions, the philosopher Daniel Dennett recommends using the "surely alarm." Whenever someone says - or thinks - "surely this is the case..." one should pay special attention to the claim being made and challenge how "sure" this is (see Figure 2). The reverse exercise can be illustrative as well: If you find yourself saying "This will never happen in our industry!" then you should assume that some startup is already working on this because it is a blind spot in the industry.
> Beware of "digital cargo cults" - market evidence must trump appearances /// Not every digital action is a valid response to digital transformation. Nevertheless, leaders might be inclined to think that they are on track because they are not familiar enough with the logic of the new system. What happens in such a case can be demonstrated very well with what are known as cargo cults. After temporary airbases in the Melanesian islands had been abandoned after the Second World War, the locals recreated the external appearance of airplanes, landing strips and control towers using wood and palm leaves in an attempt to lure back the military planes that were bringing cargo to the island. The scientist and Nobel Prize winner Richard Feynman used this example to criticize what he called cargo cult science: activities that mimic the appearance of scientific activity to the layperson. Typically, complicated terminology is used - without actually following the essence of the research object and without stringent hypothesis testing.

Companies trying to run digital transformation projects without actually putting digital talent in positions of power are running a similar risk. The result can easily be something that looks digital to the companies' top management - after all, it uses the "IOT" and has a "platform" and even a cool "app" for smartphones! But top management may not realize that under the surface, their activities lack the essence of what makes digital projects successful: creating new added value, dramatically increasing speed and ease of use, reducing complexity and lowering costs substantially. 
> Apply design thinking for rapid prototyping and a fastfailure culture /// Design thinking has moved into the corporate mainstream over the last two decades after starting out as a niche for developing new products or services. One of its core practices is a rapid iteration of simple, rough prototypes allowing enough user interaction to elicit meaningful real-world feedback: "A prototype is worth 1000 words." A key difference in the design thinking approach compared to traditional development is that failure is an accepted - and even celebrated - part of the process. Acknowledging the inherent risk in a truly new development implies making many small bets by seed funding parallel projects and accepting that most of them will not work. The key lies in then selecting and scaling those "minimal viable products" that have shown to work.

$>$ Transform decision-making to attract digital talent in time /// If you want to attract top digital talent, hierarchy can be a strong deterrent. In the 2016 Global Perspectives Barometer, about 800 "Leaders of Tomorrow" cited "hierarchy that slows down decision-making" as the second most substantial risk for established companies in today's fast-paced markets. The top risk was "innovation blindness," the inability to recognize the need for a decision and staying passive in a quickly changing environment. When it comes to good decision-making, experience from the pre-digital era is much less valuable in the eyes of the "Leaders of Tomorrow" than openness to new ideas and the ability to manage the flood of digital information. In order to keep their best employees, companies must involve them more closely than before in the decisionmaking process and delegate more decisions.

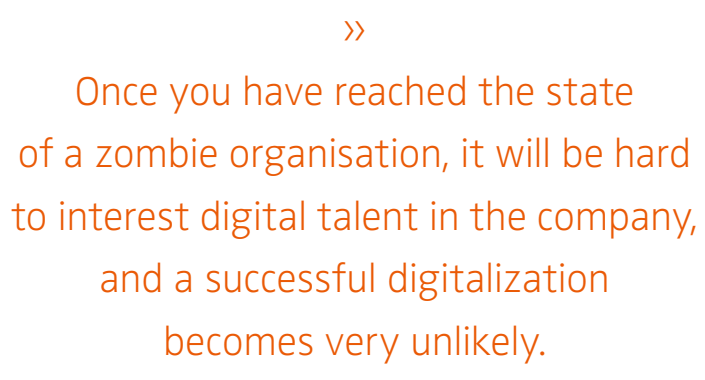

$\ll$
The future is already here - it is just not very evenly distributed /// To get your fair share of the future, do not look at digitalization as an internal technology problem. Look at its impact on your customers, your categories, your markets and transaction costs. Digitalization is a challenge because it creates new products, services and market mechanisms; eliminates barriers to entry; erodes category boundaries and violates some of the basic assumptions on which current business models are built. Therefore, learning from start-ups rather than relying on experience seems more adequate. If you can attract savvy digital natives and give them the authority to be critical, flexible and value oriented, your chances to survive or even grow in a transformed business world will still be good. Leave the zombie role to others!

\section{FURTHER READING}

Buder, F.; Neus, A.; Mueller, A.-L. (2016):

Global Perspectives Barometer 2016 -

Voices of the Leaders of Tomorrow:

Transform Decision Making!

GfK Verein and St. Gallen Symposium

Dennett, D. C. (2013):

Intuition pumps and other tools for thinking. WW Norton \& Company.

Dunning, D. (2011):

The Dunning-Kruger Effect:

On Being Ignorant of One's Own Ignorance. Advances in Experimental Social Psychology,

Vol. 44 , pp. $247-296$

Feynman, Richard P. (1974):

Cargo Cult Science. Some Remarks on science, pseudoscience, and learning how not to fool yourself. calteches.library.caltech.edu/51/2/CargoCult.htm 